\title{
The effect of local environment on photoluminescence: A time-dependent density functional theory study of silanone groups on the surface of silica nanostructures
}

\author{
M. A. Zwijnenburg, ${ }^{1}$ A. A. Sokol, ${ }^{2}$ C. Sousa, ${ }^{1}$ and S. T. Bromley ${ }^{1,3, a)}$ \\ ${ }^{1}$ Departament de Química Física and Institut de Química Teòrica i Computacional, \\ Universitat de Barcelona, 08028 Barcelona, Spain \\ ${ }^{2}$ Department of Chemistry, 3rd Floor, Kathleen Lonsdale Building, University College London, \\ Gower Street, London WC1E 6BT, United Kingdom \\ ${ }^{3}$ Institució Catalana de Recerca i Estudis Avançats (ICREA), 08010 Barcelona, Spain
}

(Received 2 April 2009; accepted 27 May 2009; published online 17 July 2009)

\begin{abstract}
The optical absorption spectrum and lowest photoluminescence (PL) signal for silanone terminated silica nanostructures are studied using time-dependent density functional theory calculations on a range of realistic low energy silica nanocluster models. We show that the broad experimental absorption spectrum for silanone centers [V. A. Radtsig and I. M. Senchenya Russ. Chem. Bull. 45, 1849 (1996)] is most likely the result of a synergetic combination of inhomogeneous broadening, thermal broadening and the small energy differences between different excitations. We further demonstrate that upon relaxation of the excited state the excited electron and hole localize on only one silanone center, and that there is a clear and distinct link between the local environment of a silanone center and its absorption and PL spectra. Finally, we provide strong evidence that the silanone center does not have a double bond between the constituent silicon and oxygen atoms but rather can be probably more aptly described as the $=\mathrm{Si}^{+}-\mathrm{O}^{-}$charge-transfer species. () 2009 American Institute of Physics. [DOI: 10.1063/1.3155083]
\end{abstract}

\section{INTRODUCTION}

A wide variety of silica $\left(\mathrm{SiO}_{2}\right)$ nanostructures have been shown to display room temperature red, ${ }^{1-5}$ green, ${ }^{3,5,6}$ blue, ${ }^{3,6-9}$ and white ${ }^{10}$ photoluminescence (PL). Silica is a wide-gap insulating material with the top of its valence band strongly localized on the oxygen sublattice. The observed PL is thus unlikely to be the result of quantum confinement ${ }^{11}$ (as is commonly assumed to be the case for semiconductor nanostructures) but rather originates from defect states within the gap. Often such defect states arise on or near the surface where silica deviates away from forming a continuous bonded network of corner-sharing $\mathrm{SiO}_{4}$ tetrahedra (i.e., four-coordinated silicon atoms linked by two-coordinated oxygen atoms) and forms other more energetically favorable structural motives with electronic signatures distinct from the bulk. As silica nanostructures inherently possess large surface area to volume ratios it is reasonable to anticipate that much of the nanospecific silica PL is due to such terminating defects.

Detailed theoretical studies have demonstrated that low energy nanosized silica clusters $\left[\left(\mathrm{SiO}_{2}\right)_{N}\right.$ with $5<N<28]^{12,13}$ are terminated with silanone groups $(=\mathrm{Si}$ $=\mathrm{O})$ and/or a distinct combination of a triply and a singly coordinated oxygen atoms (the compensated nonbridging oxygen defect ${ }^{14,15}$ ). Other higher energy terminating defects (e.g., isolated triply coordinated silicon centers, $\equiv \mathrm{Si}$ ) have also been proposed in the literature. These latter defects, tend

\footnotetext{
a) Author to whom correspondence should be addressed. Electronic mail: s.bromley@ub.edu.
}

not to be found in low energy nanoclusters, but can be thought to be formed as the result of kinetic trapping [e.g., by high barriers preventing amorphous silica exploring low(er) energy structures when quenched below its glass temperature] or high energy irradiation during preparation or measurement (e.g., as in cathodeluminescence).

To assist in the experimental identification of the defect centers responsible for particular luminescence signals, theoreticians have attempted to calculate the absorption and emission spectra for the above mentioned defects. ${ }^{16-19}$ However, as the majority of these calculations were performed using correlated wave function based methods [e.g., complete active space second-order perturbation theory (CASPT2), ${ }^{20,21}$ and multireference doubly excited configuration interaction method ${ }^{22}$ ] such studies were typically limited to very small specifically constructed hydrogen-terminated fragments. While this approach allows one to obtain accurate estimates of the intrinsic absorption and luminescence energies of an isolated defect, it does not include the effect of the local environment of a defect on its spectra. Such studies, therefore, inherently do not yield information on the width and shape of absorption and luminescence lines in amorphous or poorly crystallized samples. Herein, in contrast, we employ the computationally less expensive time-dependent density functional theory (TD-DFT) ${ }^{23,24}$ to calculate the absorption and emission spectra of realistic dry silica clusters obtained through global optimization. By taking a range of low energy cluster isomers we naturally and directly probe the influence of variations in the local environment in which the defect is embedded on the PL. This methodology exploits 
the fact that low energy $\left(\mathrm{SiO}_{2}\right)_{N}$ clusters are natural model systems of the dry surfaces of nanosilica. ${ }^{25,26}$ Specifically, we apply this approach to the study of the PL spectra of silanone terminated nanoclusters.

Experimentally silanone defects can be formed by the condensation of geminal hydroxyls on hydroxylated silica surfaces or specifically prepared via a four step route using methoxylated silica $\left(\equiv \mathrm{Si}-\mathrm{O}-\mathrm{CH}_{3}\right)$ as a starting material Radzig $^{27}$ and Radtsig and Senchenya ${ }^{28}$ ). From such investigations only a very broad absorption spectrum has thus far been reported. $^{27}$ Theoretically, the silanone absorption and luminescence spectrum of small silanone containing model molecules [e.g., $\left.\left(\mathrm{OSiH}_{3}\right)_{2} \mathrm{Si}=\mathrm{O}\right]$ were also studied by Raghavachari and Pacchioni ${ }^{29,30}$ and Zyubin et al., ${ }^{31}$ respectively. Using a range of realistic low energy silica nanocluster models we show that the broad experimental absorption spectrum for silanone centers is most likely the result of a combination of inhomogeneous broadening and the small energy differences between different excitations. We further demonstrate, that, when multiple silanone centers are present upon relaxation of the excited state, the excited electron and hole localize on only one silanone center. Furthermore, we show that there is a clear and distinct link between the local environment of a silanone center and its absorption and PL spectra. Finally, we provide strong evidence that the silanone center does not have, by analogy with the $\mathrm{C}=\mathrm{O}$ group, a double bond between the constituent silicon and oxygen atoms, but rather can be perhaps more appropriately described as the charge-transfer species $=\mathrm{Si}^{+}-\mathrm{O}^{-}$.

\section{COMPUTATIONAL DETAILS}

The luminescence spectra of silanone terminated clusters were calculated using a three step approach. In the first step the ground state geometry of each cluster was optimized using conventional DFT, in the second step the absorption spectra of the clusters were calculated using TD-DFT, while in the final step the energy of a selected excited state was minimized using TD-DFT. A critical comparison of the available literature data (including our recent work on the spectra of $\mathrm{Si}_{4} \mathrm{O}_{8}$ clusters ${ }^{14}$ ) suggests that the combination of B3LYP (Ref. 32) with a Pople 6-31G* basis set ${ }^{33,34}$ is a reasonable choice with respect to the results of wave function based methods for silanone terminated clusters. This combination of functional and basis set was therefore used in the majority of the results discussed. Results for selected small systems were further rechecked using a combination of (TD-)B3LYP with the larger aug-cc-pVTZ basis ${ }^{35,36}$ that also includes diffuse basis functions, and by CASPT2 single point calculations on (TD-)B3LYP/aug-cc-pVTZ basis optimized geometries.

All the DFT results were obtained using the TURBOMOLE 5.10 code, $^{37}$ which includes analytical forces for TD-DFT (using the Lagrangian formalism developed by Furche and co-workers ${ }^{38-40}$ ) and thus allowed us to obtain numerically accurate relaxed excited state energies. In each case ground state and excited state geometries were relaxed until the maximum norm of the Cartesian gradients in the cluster was smaller than $1 \times 10^{-5}$ Hartree/Bohr.

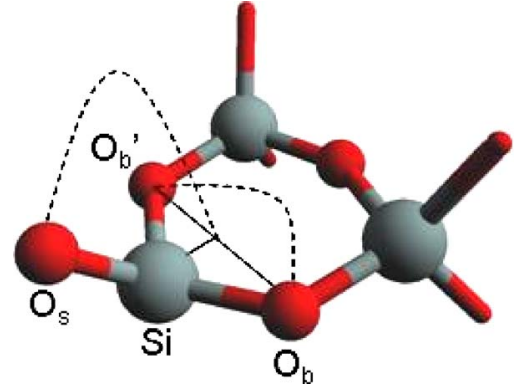

FIG. 1. Fragment of the $\mathrm{Si}_{16} \mathrm{O}_{32}$ cluster showing the structural degrees of freedom around the silanone center. Shown is a typical relaxed excited state configuration.

The ground and low-lying excited states of selected small clusters were also calculated using the CASPT2 method as implemented in the MOLCAS-7 package. ${ }^{41}$ In this approach, first an $N$-electron complete active space selfconsistent field (CASSCF) wave function is computed, which includes an important part of the electron correlation in a variational way, and in a second step, the remainder, mainly dynamical electron correlation, is obtained by a second-order perturbational treatment with the CASSCF wave function as zeroth-order wave function. This strategy has been successfully applied to study excited states in solidstate compounds ${ }^{42-44}$ including the study of the optical spectra of point defects in silica. ${ }^{45}$ The basis sets used in the CASPT2 calculations for all atoms is the augmented aug-ccpVTZ basis. ${ }^{35,36}$ The CASSCF wave functions were constructed according to the symmetry point groups of the systems. To define the active space, restricted active space SCF calculations have been performed and the orbitals with the largest deviation from 0 or 2 in the natural orbital occupation numbers have been included in the active space. Thus, the final active space included 12 orbitals and 12 electrons where all valence electrons of the silanone groups ( $\mathrm{Si} 3 s, 3 p$ and $\mathrm{O}$ $2 s, 2 p)$ have been correlated in the CASPT2 calculation.

\section{RESULTS AND DISCUSSION}

As a first step we geometry optimized the ground states for a set of low energy silica clusters terminated exclusively with silanone groups and subsequently calculated their vertical absorption spectra. This set included the lowest energy isomers for $\mathrm{Si}_{4} \mathrm{O}_{8}, \mathrm{Si}_{6} \mathrm{O}_{12}, \mathrm{Si}_{8} \mathrm{O}_{16}, \mathrm{Si}_{10} \mathrm{O}_{20}, \mathrm{Si}_{14} \mathrm{O}_{28}, \mathrm{Si}_{16} \mathrm{O}_{32}$, $\mathrm{Si}_{21} \mathrm{O}_{42}$, and $\mathrm{Si}_{23} \mathrm{O}_{46}$ (see Refs. 12 and 13) together with the second lowest energy isomer for $\mathrm{Si}_{6} \mathrm{O}_{12}$ (which is nearly degenerate in energy with the lowest energy cluster for $\mathrm{Si}_{6} \mathrm{O}_{12}$ ). This set of clusters employed allows us to sample a large range of environments around the silanone (e.g., $\mathrm{O}_{b}-\mathrm{Si}-\mathrm{O}_{b}{ }^{\prime}$ $89.5^{\circ}-115^{\circ}$, see Fig. 1 for notation). While this set by no means covers all possible environments a silanone groups could reside in on a dry silica surface; it allows for an efficient first assessment of the influence of local environment on the electronic spectra of silanone groups. The TD-DFT calculated absorption spectra up to $6.5 \mathrm{eV}$ for five representative clusters in the set are presented in Fig. 2 and show that the onset of absorption $(\mathrm{S} 0 \rightarrow \mathrm{S} 1)$ lies between 4.7 and 5.1 $\mathrm{eV}$ (depending on the cluster in question). This onset does not change with increasing the basis-set quality beyond 


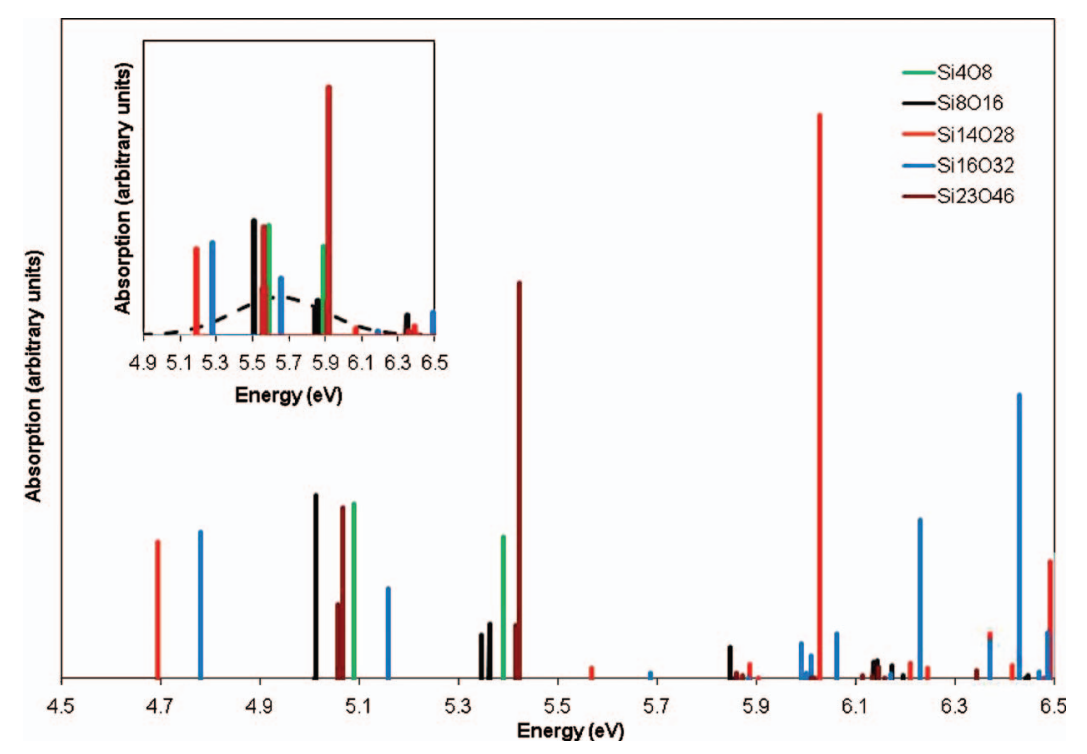

FIG. 2. TD-DFT calculated absorption spectra up to 6.5 $\mathrm{eV}$ for five representative silica clusters. The inset shows the same spectra shifted upwards in energy by $0.5 \mathrm{eV}$ inline with that suggested by CASPT2 results on $\mathrm{Si}_{4} \mathrm{O}_{8}$. The experimentally measured spectrum (Ref. 27) is represented by a dashed Gaussian in the inset with parameters chosen to match the reported experimental peak maximum and FWHM.
6-31G*, as calculations with a aug-cc-pVTZ basis on the four smallest clusters (for which the geometry was also reoptimized) show that the lowest excitation energies for each cluster are consistently shifted downwards by only $0.02-0.1$ eV. Finally, a CASPT2 calculation on the B3LYP/aug-ccpVTZ optimized $\mathrm{Si}_{4} \mathrm{O}_{8}$ cluster yields an absorption onset of 5.56 (compared with 5.04 for TD-B3LYP/aug-cc-pVTZ), suggesting that the TD-B3LYP results may be approximately $0.5 \mathrm{eV}$ (or $10 \%$ ) too low. The latter difference is in line with the general trend observed in an extensive comparison of TD-B3LYP and wave-function methods for excitation energies of silica defects. ${ }^{30}$

The previously reported TD-B3LYP absorption onsets of the $\left(\mathrm{OSiH}_{3}\right)_{2} \mathrm{Si}=\mathrm{O}$ molecule (5.09 and $5.12 \mathrm{eV}$ in Refs. 31 and 30, respectively) lie near the top of the range obtained herein. Specifically, comparing with our results, we find that these values are similar to the absorption onsets in those clusters in which the silanone center is attached to a twomembered (i.e., $\mathrm{Si}_{2} \mathrm{O}_{2}$ ) ring. This can be understood as the local geometry around the silanone center in the $\left(\mathrm{OSiH}_{3}\right)_{2} \mathrm{Si}=\mathrm{O}$ molecule (e.g., an $\mathrm{O}_{b}-\mathrm{Si}-\mathrm{O}_{b}{ }^{\prime}$ angle of $105.7^{\circ}$ as reported in Ref. 31) lies in between that exhibited by the two- and three-membered (i.e., $\mathrm{Si}_{3} \mathrm{O}_{3}$ ) rings in the clusters in our study.

It is interesting to compare our calculated data with the experimental absorption spectrum of silanone groups prepared on the surface of amorphous silica, for which Radzig ${ }^{27}$ reports an absorption band with broad maximum at $5.65 \pm 0.1 \mathrm{eV}$ with a full width at half maximum (FWHM) of $\sim 0.9 \mathrm{eV}$ (represented by the dashed line in the inset in Fig. 2). While the very large broadening of the experimental band makes it difficult to match our computed absorption bands to the one observed in experiment, we find that the experimental onset of absorption lies in the same range as predicted by our calculations. This agreement is particularly striking if the calculated bands are shifted upwards by 0.5 $\mathrm{eV}$, as is suggested by the CASPT2 result (see inset in Fig. 2). Between the absorption onset and $6.5 \mathrm{eV}$ there are several strong intensity absorption peaks. The presence of more than one strong intensity absorption peak (even when considering only a single defect) within the experimental peak width, strongly suggests that the experimental broadening is not merely the result of conventional inhomogeneous broadening (i.e., whereby defect centers have slightly different local environments resulting in a distribution of absorption energies and an averaged absorption spectrum ${ }^{46,47}$ ) and thermal broadening, but is also due to the overlap of the individual broadened peaks.

We have further estimated the expected life-time $\tau$ of the lowest excited state of the clusters at their ground state geometry from the calculated oscillator strength $f$ (using $\tau$ $=c^{2} /\left(2 E_{\mathrm{abs}}^{2} f\right)$, where $E_{\mathrm{abs}}$ is the absorption energy and $c$ the speed of light). The obtained life-time estimates (e.g., for the $\mathrm{Si}_{4} \mathrm{O}_{8}$ chain: $22 \mathrm{~ns}$ for TD-DFT/6-31G* $32 \mathrm{~ns}$ for TD-DFT/ aug-cc-pVTZ, and 9 ns with CASPT2/aug-cc-pVTZ) are more than two orders of magnitude larger than the characteristic time-scale of a low energy phonon $\left(\sim 1 \mathrm{~cm}^{-1}\right.$ corresponding to $\sim 33 \mathrm{ps}$ ). This suggests that after excitation, either directly or by trickling down from higher excited states, the lowest excited singlet state lives long enough for the system to relax toward one of the minima on the first singlet excited state surface before collapsing back to the ground state. Luminescence, therefore most likely will take place in a Frank-Condon fashion vertically from the excited state singlet minima (S1 state at the minimal energy geometry for the $\mathrm{S} 1$ state: $\mathrm{S} 1 / \mathrm{S} 1_{\min }$ ) back to the ground state surface (S0 state at the minimal energy geometry for the $\mathrm{S} 1$ state: $\mathrm{S} 0 / \mathrm{S} 1_{\text {min }}$ ) and at a lower energy than at absorption. (Note: In principle a lower energy defect state could lie across a barrier on the ground state potential energy surface, which would result in a more complex process of photostimulated defect formation; however, no such states were found in this study.) The latter red shift, often referred to as Stokes shift, is a direct result of the relaxation in the excited state and its calculation is what we will focus on in the remainder of this paper.

To study the relaxation of a given excited state of a particular cluster, the geometry of the excited state was optimized using TD-DFT yielding the geometry of the excited state minimum, the vertical emission energy at the excited state minimum and the Stokes shift (as the difference be- 


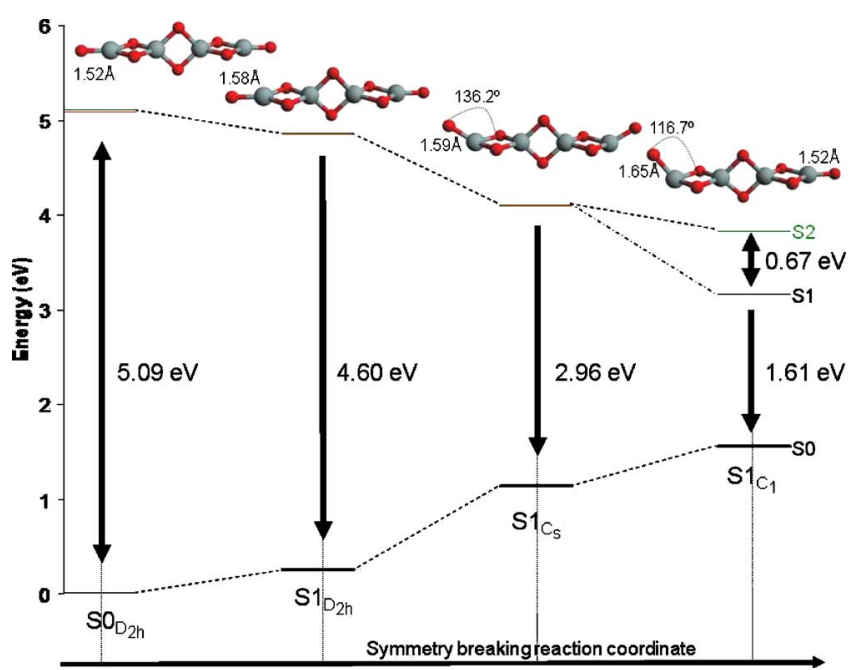

FIG. 3. Stationary points studied for the $\mathrm{Si}_{4} \mathrm{O}_{8}$ cluster as a function of the symmetry breaking reaction coordinate.

tween the vertical absorption energy at the ground state geometry and the vertical emission energy at the excited state minimum). For the smaller clusters this optimization step was followed by a numerical frequency calculation on the obtained structure to verify that it indeed corresponded to a minimum on the excited state energy landscape. This latter confirmation is not necessarily trivial and can be seen from the example of the $\mathrm{Si}_{4} \mathrm{O}_{8}$ chain in Fig. 3. The ground state of the $\mathrm{Si}_{4} \mathrm{O}_{8}$ chain has $\mathrm{D}_{2 \mathrm{~h}}$ symmetry, and when the lowest excited singlet state is optimized in this point group the result is a stationary point with five imaginary frequencies (see Fig. 3, point $\left.\mathrm{S} 1_{\mathrm{D} 2 \mathrm{~h}}\right)$. When the structure is reoptimized starting from point $S 1_{D 2 h}$ and the point group symmetry constraint is relaxed from $D_{2 h}$ to $C_{s}$ (where also manually the two terminal oxygen atoms are moved away by a finite but small amount from the twofold axis running down the length of the cluster), a lower energy stationary point with six imaginary frequencies (see Fig. 3, point $S 1_{\mathrm{Cs}}$ ) is obtained. Finally, a re-optimization step starting from point $S 1_{C s}$ without any symmetry constraints results in the proper minimum (see Fig. 3, point $\mathrm{S}_{\mathrm{C} 1}$ ). The $\mathrm{Si}_{4} \mathrm{O}_{8}$ chain cluster thus is found to break symmetry completely upon relaxation down the excited state energy surface. In this context especially the latter symmetry breaking from $\mathrm{C}_{\mathrm{s}}$ to $\mathrm{C}_{1}$ is interesting, as while in $\mathrm{C}_{\mathrm{s}}$ (as for $\mathrm{D}_{2 \mathrm{~d}}$ ) both terminal silanone groups are symmetrically equivalent this equivalence is lost in $\mathrm{C}_{1}$. This loss of equivalence has important implications as it is associated with a complete localization of the excited electron and hole on one of the two silanone groups (as probed by the Mulliken charges for the excited state), where the silanone bond on which the electron localizes elongates to $1.65 \AA$ while the other silanone shortens back to its length in the ground state. The mechanism underlying the symmetry breaking appears to be pseudo-Jahn-Teller ${ }^{48,49}$ like in nature, where the splitting of the two highest occupied molecular orbitals of the ground state $(32 \mathrm{meV})$ is very small compared with the energy lowering associated with the symmetry breaking distortion $(1.35 \mathrm{eV}$ ). All other clusters studied (bar $\mathrm{Si}_{21} \mathrm{O}_{42}$ ) also have symmetrically equivalent silanone groups in the ground state, and are found to undergo a similar symmetry breaking upon relaxation.

The TD-B3LYP/6-31G* calculated PL energies and Stokes shifts from the lowest singlet excited states span narrow ranges of 1.48-1.61 eV and 3.22-3.55 eV, respectively (see Fig. 4). These calculations thus show that the effect of the environment on the PL peak of the silanone defect is small and that it will be inherently much sharper than the corresponding absorption peak. TD-B3LYP/aug-cc-pVTZ calculations on the four smallest clusters (in which the structures were reoptimized using the larger basis set) do not change this observation, as they yield only a minor shift upwards $(0.07-0.1 \mathrm{eV})$ upon increasing the basis-set quality. Finally, CASPT2 calculation on the TD-B3LYP/aug-ccpVTZ optimized $\mathrm{Si}_{4} \mathrm{O}_{8}$ cluster gives a PL energy for the lowest excited singlet state of $1.61 \mathrm{eV}(6 \%$ lower than the $1.71 \mathrm{eV}$ obtained for TD-B3LYP/aug-cc-pVTZ) and reinforces our trust in the reliability of the TD-B3LYP results.

Our obtained PL energies agree well with the PL energy obtained for the lowest excited state of the $\left(\mathrm{OSiH}_{3}\right)_{2} \mathrm{Si}=\mathrm{O}$ molecule $(1.58 \mathrm{eV})$ by Zyubin et al. ${ }^{31}$ using TD-B3LYP and a manual and partial (i.e., only considering one angle and the silanone bond length) optimization of the excited state geometry starting from the geometry of the lowest triplet state.

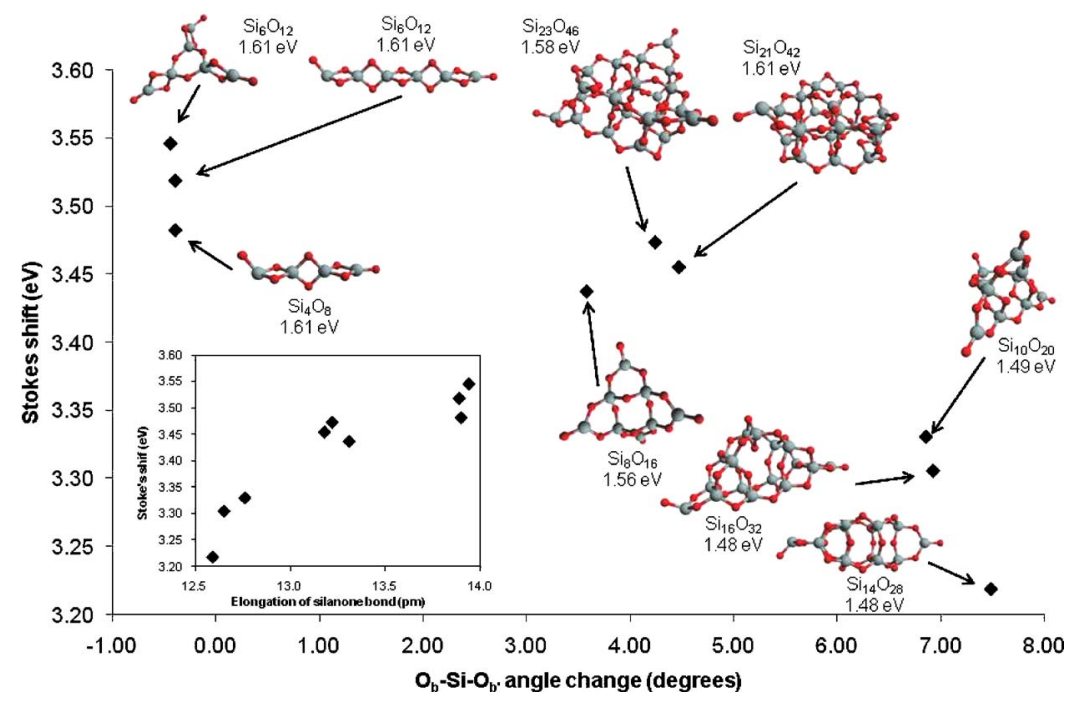

FIG. 4. Stokes shift in the lowest singlet excited states of the silanone terminated clusters as a function of the change in the $\mathrm{O}_{b}-\mathrm{Si}-\mathrm{O}_{b}{ }^{\prime}$ angle upon relaxation of the excited state. All structures are additionally labeled with their calculated PL energy. The inset shows the Stokes shift as a function of the elongation of the silanone bond. 
Moreover, a CASPT2 calculation by Zyubin et al. ${ }^{31}$ on the CASPT2 optimized geometry of the second lowest excited singlet state of the $\left(\mathrm{OSiH}_{3}\right)_{2} \mathrm{Si}=\mathrm{O}$ molecule (which most likely is very similar in structure to the lowest excited singlet state) yields an excitation energy for the lowest exited singlet state of $1.63 \mathrm{eV}$ which also fits well with the results obtained herein.

As discussed above and as can be seen in Fig. 3 the relaxation of the lowest singlet excited state of a silanone terminated cluster is associated with a number of structural changes. First, the silanone bond on which the excited electron localizes elongates. Second, there is a bending of the $\mathrm{Si}-\mathrm{O}_{s}$ bond out of the plane defined by the silicon atom and the two doubly coordinated oxygen $\left(\mathrm{O}_{b}\right.$ and $\left.\mathrm{O}_{b}{ }^{\prime}\right)$ atoms bonded to it (see Fig. 1 for definitions of the relevant angles and distances). Furthermore, upon relaxation the $\mathrm{O}_{b}-\mathrm{Si}-\mathrm{O}_{b}{ }^{\prime}$ angle also shows a significant change toward larger values and overall the geometry around the silicon changes from trigonal to trigonal pyramidal [which fits with the observation of Zyubin et $a l^{31}$ for the manually optimized $\left(\mathrm{OSiH}_{3}\right)_{2} \mathrm{Si}=\mathrm{O}$ molecule].

Figure 4 demonstrates that even though the PL energies of the different clusters span a narrow range, there is nevertheless a strong correlation between the local environment of the silanone center and its predicted Stokes shift. The larger the change in the $\mathrm{O}_{b}-\mathrm{Si}-\mathrm{O}_{b}{ }^{\prime}$ angle is upon relaxation of the excited state, the smaller is the Stokes shift. In other words the larger the mechanical flexibility of the cluster around the silanone center is, the smaller is the energy gained by relaxation. In contrast, the elongation of the silanone bond $\left(\mathrm{Si}-\mathrm{O}_{s}\right)$, which is not in any way mechanically constrained by the remainder of the cluster, is maximal for the clusters with the largest Stokes shift and seems to be anticorrelated with the change in the $\mathrm{O}_{b}-\mathrm{Si}-\mathrm{O}_{b}{ }^{\prime}$ angle. This effect is so pronounced that one could argue that if a cluster cannot relax sufficiently by enlarging the $\mathrm{O}_{b}-\mathrm{Si}-\mathrm{O}_{b}{ }^{\prime}$ angle it attempts to relax instead by elongating the silanone bond further than it would have done otherwise.

Finally, it should be noted here that the clusters with the largest Stokes shift are not, as perhaps expected, the clusters with the lowest $\mathrm{PL}$ energy. The change in $\mathrm{O}_{b}-\mathrm{Si}-\mathrm{O}_{b}{ }^{\prime}$ angle upon relaxation of the excited state is positively correlated with the ground state $\mathrm{O}_{b}-\mathrm{Si}-\mathrm{O}_{b}{ }^{\prime}$ angle; the larger the $\mathrm{O}_{b}-\mathrm{Si}-\mathrm{O}_{b}{ }^{\prime}$ angle the more flexible the structure is around the silanone center. The Stokes shift increases upon decreasing the flexibility of the structure (see above), thus the Stokes shift is negatively correlated with the size of the ground state $\mathrm{O}_{b}-\mathrm{Si}-\mathrm{O}_{b}{ }^{\prime}$ angle. Moreover, the lowest absorption energies of the clusters (the absorption onset) also decrease with the ground state $\mathrm{O}_{b}-\mathrm{Si}-\mathrm{O}_{b}{ }^{\prime}$ angle. As the $\mathrm{PL}$ energy equals the absorption energy minus the Stokes shift, and as the decrease in the Stokes shift is smaller than the lowering of the absorption energy with respect to the ground state $\mathrm{O}_{b}-\mathrm{Si}-\mathrm{O}_{b}{ }^{\prime}$ angle, the PL energy tends to decrease with the ground state $\mathrm{O}_{b}-\mathrm{Si}-\mathrm{O}_{b}{ }^{\prime}$ angle.

A possible driving force for the Stokes shift and associated geometry changes can be seen from a closer inspection of the orbitals involved in the absorption and PL. Figure 5 shows that upon absorption of a photon an electron is excited

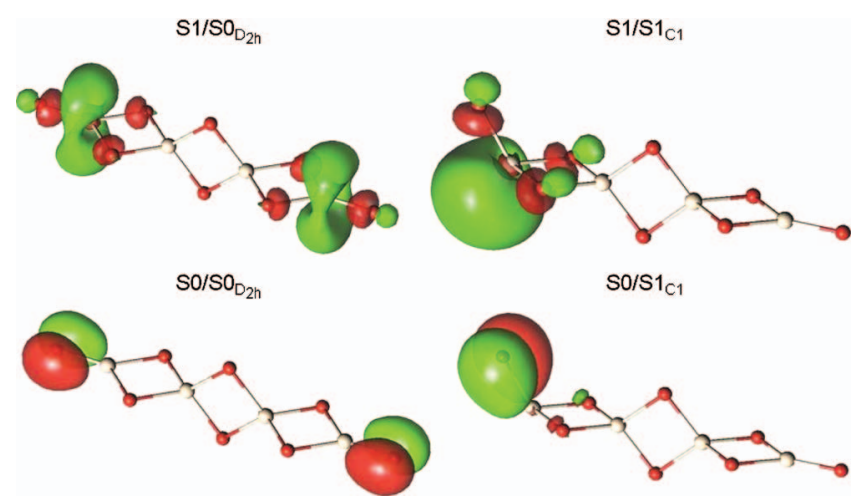

FIG. 5. Molecular orbitals as obtained from an average of all configurations from the relevant energy levels in the CASSCF calculations corresponding to the transferred electron and remaining hole for the $\mathrm{S}_{\mathrm{D} 2 \mathrm{~h}}$ and $\mathrm{S}_{\mathrm{C} 1}$ geometries of $\mathrm{Si}_{4} \mathrm{O}_{8}$.

from a nonbonding orbital on the oxygen atom of the silanone defect (corresponding to an $\mathrm{O} 2 p$ like lone-pair) into an antibonding $\sigma^{*}$ orbital of the silanone bond which has as largest contributor the silicon $4 s$ orbital. The PL, however, takes places from a nonbonding orbital located on the same silicon atom (corresponding to a $\mathrm{Si} s p^{3}$-hybrid like lone-pair) back to a nonbonding orbital on the pendant oxygen atom. Furthermore, a critical analysis of the ground state molecular orbitals strongly suggests that in the ground state geometry there is no double bond between the silicon and oxygen atoms that form the silanone center. B3LYP and CASSCF results show no evidence for a $\pi$ bond between silicon and oxygen. In contrast, we observe that the silicon donates its $p_{z}$ electron to the pendant $\mathrm{O}$ and that the bonding in the silanone center is perhaps better described as $=\mathrm{Si}^{+}-\mathrm{O}^{-}$instead of $\mathrm{Si}=\mathrm{O}$. This classification, which differs from that commonly used in the literature (in which the silanone is assumed to be analogous to a carbonyl $\mathrm{C}=\mathrm{O}$ center), is consistent with the short bond length of the silanone [1.51-1.52 $\AA$, slightly shorter than that found for the siloxy anion $\equiv \mathrm{Si}-\mathrm{O}^{-}, \sim 1.55 \AA$ (Refs. 50 and 51)], the more electropositive nature of $\mathrm{Si}$ compared to $\mathrm{C}$ and the substantial ionic nature of bonding in silica. ${ }^{52}$ Moreover, this interpretation is also consistent with the notable difference in reactivity between silanone and carbonyl groups. The silanone center is known to be extremely reactive with respect to nucleophilic attack by water, ${ }^{53,54}$ while the carbonyl group (e.g., in formaldehyde) is virtually unreactive under similar conditions.

Based on this information we suggest a set of events occurring upon absorption of a photon. First, an electron is transferred away from the oxygen in the silanone center toward the silicon. This electron transfer results in the formation of a hole in the oxygen nonbonding orbital that the electron was excited from and thus the formation of what is effectively a close analog of a nonbridging oxygen hole center $(\mathrm{NBOHC})(\equiv \mathrm{Si}-\mathrm{O} \cdot)$. This process in conjunction with the occupation by the excited electron of an antibonding $\sigma^{*}$ orbital, destabilizes the silanone bond and leads to its elongation to a length (1.64-1.65 $\AA$ ) that approaches that typical of a NBOHC $[\sim 1.66-1.69 \AA$ (Refs. 50 and 51)]. Finally, the excited electron orbital converts into a $s p^{3}$ hybridlike nonbonding orbital on the silicon atom (much like $\equiv \mathrm{Si}$, the 
surface $E^{\prime}$ center ${ }^{19,55}$ ), and the cluster relaxes away from the original planar symmetry to minimize repulsion between the three oxygen atoms around the silicon center and its now partly occupied non-bonding orbital. The large Stokes shift $(3.22-3.55 \mathrm{eV})$ observed for the silanone defects thus seems to stem directly from the significant redistribution of electron density upon excitation $\left(=\mathrm{Si}^{+}-\mathrm{O}^{-} \rightarrow=\mathrm{Si} \cdot-\mathrm{O} \cdot\right)$ and the geometry changes that this electron transfer induces.

Based on our theoretical results presented above, we believe that it would be very interesting to repeat the original experiments of Radtsig and Senchenya ${ }^{28}$ using a welldefined silica surfaces as obtained in surface science experiments (e.g., by studying the optical spectra of quartz single crystals after conversion of their surface silanols into silanone groups). Such investigations, in combination with advanced spectroscopic techniques such as hole burning spectroscopy $^{56}$ and/or site-selective luminescence spectroscopy, ${ }^{57}$ would both yield a better resolved absorption spectrum (e.g., as a result of reduced inhomogeneous broadening due to increased uniformity of surface sites) to compare with our predictions, and provide experimental evidence for the predicted PL signal.

\section{CONCLUSIONS}

The optical absorption spectrum and lowest PL signal for silanone terminated nanosized silica are studied using TDDFT calculations on realistic low energy silica clusters. In doing so we exploit the fact that such silica clusters are natural model systems of the dry surfaces of nanosilica and can yield information on the influence of the local environment of a defect. In particular, we stress that such an approach allows for insights that are unattainable with the small specifically constructed hydrogen-terminated fragments more typically employed to study theoretically optical excitations in materials. Our calculations strongly suggest that the very broad absorption peak measured in experiment for silanone centers on the surface of silica most likely results from a synergistic combination of inhomogeneous broadening, thermal broadening and the small differences in energy between different excitations. Silanone centers that are symmetrically equivalent for the ground state geometry are found to contribute in equal amounts to excitations from the ground state, but we observe that this symmetry is broken upon relaxation of the excited state. The excited electron and hole localize on only one silanone center and most if not all of the original symmetry of the cluster is lost. We observe that while the calculated lowest PL signals for the different clusters span a narrow range $(1.48-1.61 \mathrm{eV})$ there is nevertheless a clear and distinct link between the local environment of a silanone center and the position of the lowest PL signal. The weaker the mechanical constraint imposed on the silanone center by the remainder of the cluster, the lower the PL energy. Finally, we show that the large Stokes shift observed for the silanone center $(3.22-3.55 \mathrm{eV})$ finds its origin in the fact that the silanone center does not contain a double bond but rather consists of a positively charged silicon atom and a negatively charged oxygen atom, between which an electron is transferred upon excitation. We envisage that the methodology followed in our study is likely to be beneficial for the study and interpretation of the optical properties of localized defect centers in other inorganic systems.

\section{ACKNOWLEDGMENTS}

We kindly acknowledge Professor J. Giorgi, Professor F. Illas, Professor A. Schluger, Professor P. Sushko, and the participants of the Benasque TDDFT 2008 summer school for stimulating discussion. M.A.Z. acknowledges the Spanish Ministry for Science and Innovation for a Juan de la Cierva fellowship. This study has further been supported by the Spanish Ministry for Science and Innovation (Grant No. FIS2008-02238), by the Generalitat de Catalunya (Grant No. 2009-SGR-1041) by the COST-D41 action and the XRQTC. Computational time on the computers of the Centre de Supercomputació de Catalunya (CESCA) is gratefully acknowledged.

${ }^{1}$ Yu. D. Glinka, A. S. Zyubin, A. M. Mebel, S. H. Lin, L. P. Hwang, and Y. T. Chen, Eur. Phys. J. D 16, 279 (2001).

${ }^{2}$ A. S. Zyubin, Yu. D. Glinka, A. M. Mebel, S. H. Lin, L. P. Hwang, and Y. T. Chen, J. Chem. Phys. 116, 281 (2002).

${ }^{3}$ Yu. D. Glinka, S. H. Lin, and Y. T. Chen, Phys. Rev. B 66, 035404 (2002).

${ }^{4}$ A. Colder, F. Huisken, E. Trave, G. Ledoux, O. Guillois, C. Reynaud, H. Hofmeister, and E. Pippel, Nanotechnology 15, L1 (2004).

${ }^{5}$ Y. Yang, B. K. Tay, X. W. Sun, H. M. Fan, and Z. X. Shen, Physica E (Amsterdam) 31, 218 (2006).

${ }^{6}$ M. Zhang, E. Ciocan, Y. Bando, K. Wada, L. L. Cheng, and P. Pirouz, Appl. Phys. Lett. 80, 491 (2002).

${ }^{7}$ Yu. D. Glinka, S. H. Lin, and Y. T. Chen, Phys. Rev. B 62, 4733 (2000).

${ }^{8}$ Y. Hao, G. Meng, C. Ye, and L. Zhang, Appl. Phys. Lett. 87, 033106 (2005).

${ }^{9}$ A. Aboshi, N. Kurumoto, T. Yamada, and T. Uchino, J. Phys. Chem. C 111, 8483 (2007).

${ }^{10}$ T. Uchino and T. Yamada, Appl. Phys. Lett. 85, 1164 (2004).

${ }^{11}$ A. D. Yoffe, Adv. Phys. 50, 1 (2001).

${ }^{12}$ E. Flikkema and S. T. Bromley, J. Phys. Chem. B 108, 9638 (2004).

${ }^{13}$ S. T. Bromley and E. Flikkema, Phys. Rev. Lett. 95, 185505 (2005).

${ }^{14}$ M. A. Zwijnenburg, C. Sousa, A. A. Sokol, and S. T. Bromley, J. Chem. Phys. 129, 014706 (2008).

${ }^{15}$ S. Hamad and S. T. Bromley, Chem. Commun. (Cambridge) 4156 (2008).

${ }^{16}$ G. Pacchioni and G. Ierano, Phys. Rev. Lett. 79, 753 (1997).

${ }^{17}$ G. Pacchioni and G. Ierano, Phys. Rev. B 56, 7304 (1997).

${ }^{18}$ G. Pacchioni and G. Ierano, Phys. Rev. B 57, 818 (1998).

${ }^{19}$ G. Pacchioni, G. Ierano, and A. M. Marquez, Phys. Rev. Lett. 81, 377 (1998).

${ }^{20}$ K. Andersson, P. A. Malmqvist, B. O. Roos, A. J. Sadlej, and K. Wolinski, J. Phys. Chem. 94, 5483 (1990).

${ }^{21}$ K. Andersson, P. A. Malmqvist, and B. O. Roos, J. Chem. Phys. 96, 1218 (1992).

${ }^{22}$ R. J. Buenker and S. D. Peyerimhoff, Theor. Chim. Acta 35, 33 (1974).

${ }^{23}$ E. Runge and E. K. U. Gross, Phys. Rev. Lett. 52, 997 (1984).

${ }^{24}$ E. K. U. Gross and K. Burke, Lect. Notes Phys. 706, 1 (2006).

${ }^{25}$ L. S. Wang, J. B. Nicholas, M. Dupuis, H. Wu, and S. D. Colson, Phys. Rev. Lett. 78, 4450 (1997).

${ }^{26}$ S. T. Bromley, M. A. Zwijnenburg, and Th. Maschmeyer, Surf. Sci. 539, L554 (2003).

${ }^{27}$ V. A. Radzig, Colloids Surf., A 74, 91 (1993).

${ }^{28}$ V. A. Radtsig and I. M. Senchenya, Russ. Chem. Bull. 45, 1849 (1996).

${ }^{29}$ K. Raghavachari and G. Pacchioni, J. Chem. Phys. 114, 4657 (2001).

${ }^{30}$ K. Raghavachari and G. Pacchioni, J. Chem. Phys. 116, 825 (2002).

${ }^{31}$ A. S. Zyubin, A. M. Mebel, S. H. Lin, and Y. D. Glinka, J. Chem. Phys. 116, 9890 (2002).

${ }^{32}$ A. D. Becke, J. Chem. Phys. 98, 5648 (1993).

${ }^{33}$ P. C. Hariharan and J. A. Pople, Theor. Chim. Acta 28, 213 (1973).

${ }^{34}$ M. M. Francl, W. J. Petro, W. J. Hehre, J. S. Binkley, M. S. Gordon, D. J. deFrees, and J. A. Pople, J. Chem. Phys. 77, 3654 (1982). 
${ }^{35}$ T. H. Dunning, Jr., J. Chem. Phys. 90, 1007 (1989).

${ }^{36}$ D. E. Woon and T. H. Dunning, Jr., J. Chem. Phys. 98, 1358 (1993).

${ }^{37}$ R. Ahlrichs, M. Baer, M. Haeser, H. Horn, and C. Koelmel, Chem. Phys. Lett. 162, 165 (1989).

${ }^{38}$ F. Furche and R. Ahlrichs, J. Chem. Phys. 117, 7433 (2002).

${ }^{39}$ F. Furche and R. Ahlrichs, J. Chem. Phys. 121, 12772 (2004).

${ }^{40}$ D. Rappoport and F. Furche, Time Dependent Density Functional Theory, Lecture Notes in Physics (Springer, Berlin, 2006), Vol. 706.

${ }^{41}$ G. Karlström, R. Lindh, P.-A.. Malmqvist, B. O. Roos, U. Ryde, V. Veryazov, P.-O. Widmark, M. Cossi, B. Schimmelpfennig, P. Neogrady, and L. Seijo, Comput. Mater. Sci. 28, 222 (2003).

${ }^{42}$ C. de Graaf, R. Broer, and W. C. Nieuwpoort, Chem. Phys. 208, 35 (1996).

${ }^{43}$ C. de Graaf and R. Broer, Phys. Rev. B 62, 702 (2000).

${ }^{44}$ C. Sousa, C. de Graaf, F. Illas, M. T. Barriuso, J. A. Aramburu, and M. Moreno, Phys. Rev. B 62, 13366 (2000).

${ }^{45}$ C. Sousa, C. de Graaf, and G. Pacchioni, J. Chem. Phys. 114, 6259 (2001).
${ }^{46}$ A. M. Stoneham, Rev. Mod. Phys. 41, 82 (1969).

${ }^{47}$ A. B. Myers, Annu. Rev. Phys. Chem. 49, 267 (1998).

${ }^{48}$ U. Opik and M. H. L. Pryce, Proc. R. Soc. London, Ser. A 238, 425 (1957).

${ }^{49}$ A. M. Stoneham and M. Lannoo, J. Phys. Chem. Solids 30, 1769 (1969).

${ }^{50}$ T. Suzuki, L. Skuja, K. Kajihara, M. Hirano, T. Kamiya, and H. Hosono, Phys. Rev. Lett. 90, 186404 (2003).

${ }^{51}$ L. Giordano, P. V. Sushhko, G. Pacchioni, and A. L. Shluger, Phys. Rev. Lett. 99, 136801 (2007).

${ }^{52}$ M. A. Zwijnenburg, S. T. Bromley, C. Van Alsenoy, and T. Maschmeyer, J. Phys. Chem. A 106, 12376 (2002).

${ }^{53}$ F. Zhou and J. D. Head, J. Phys. Chem. B 104, 9981 (2000).

${ }^{54}$ Y. Ma, A. S. Foster, and R. M. Nieminen, J. Chem. Phys. 122, 144709 (2005).

${ }^{55}$ V. A. Radtzig, Chem. Phys. Rep. 14, 1206 (1995).

${ }^{56}$ S. Völker, Annu. Rev. Phys. Chem. 40, 499 (1989).

${ }^{57}$ L. Vaccaro, M. Cannas, and V. Radzig, Phys. Rev. B 78, 233408 (2008). 